\title{
CAMPO DE BATALLA DE LA FILOSOFÍA: ATOMISMO LACANIANO Y LA APUESTA EN TORNO AL OBJETO A *
}

\author{
Battlefield of philosophy: lacanian atomism and the bet around object petit a \\ Claudio Reyes Lozano \\ Universidad de Chile, Santiago, Chile \\ c193@hotmail.com
}

\section{Resumen}

¿En qué momento surge el vacío en la filosofía? ¿Cuándo emerge como un objeto filosófico digno de reflexión? Bajo el telón de fondo de estas preguntas propongo una tesis inicial para comenzar este trabajo: en el principio, quizás ahora también, la filosofía intentó liberarse del problema del vacío. La afirmación filosófica es una afirmación política que da cuenta de un campo de batalla: el ser es un arma que se debe usar contra un enemigo insondable. El logos, como instrumento fundamental en esta batalla, debe ser afirmado constantemente para que no caiga en la trampa de la negatividad. El problema del clinamen es indicativo de esta batalla respecto al vacío, el ser y el materialismo: Hegel hablaba despreciativamente de él; por otro lado, Deleuze realizó una formulación formidable apoyado en la causa atomista de Lucrecio explicitando una política materialista. Por último, con Lacan observamos el invento de Demócrito del den: esta produce un nuevo objeto que, sin embargo, yace materialistamente bajo desde los albores de la filosofía. Este es quizás el lugar que puede ocupar el psicoanálisis en la discusión materialista: mostrar aquel objeto, objeto $a$, como nueva herramienta en la batalla.

Palabras clave: vacío, ser, movimiento, materialismo, clinamen.

\begin{abstract}
At what moment does the emptiness appear in philosophy? When does it emerge as a philosophical object worthy of reflection? Under the backdrop of these questions I propose an initial thesis to begin this work: in the beginning, perhaps now also, philosophy tried to free itself from the problem of emptiness. Philosophical affirmation is a political statement that accounts for a battlefield: being is a weapon that must be used against an unfathomable enemy. The logos, as a fundamental instrument in this battle, must be constantly affirmed so that it does not fall into the trap of negativity. The problem of the clinamen is indicative of this battle over emptiness, being and materialism: Hegel spoke contemptuously of him; on the other hand, Deleuze made a formidable formulation based on the atomistic cause of Lucrecio, explaining a materialist policy. Finally, with Lacan we observe the invention of Democritus of the den: it produces a new object that, nevertheless, lies materialistically low since the dawn of philosophy. This is perhaps the place that psychoanalysis can occupy in the materialist discussion: to show that object, objet petit $a$, as a new tool in battle.
\end{abstract}

Keywords: emptiness, being, movement, materialism, clinamen

Fecha de Recepción: 14/06/2019 - Fecha de Aceptación: 19/12/2019

\footnotetext{
"Este ensayo es fruto de una investigación para el Seminari“Hacia un materialismo radical: Génesis mítica de lo político" dictado por el prof. Miguel Ruiz el segundo semestre de 2017 del Doctorado en Filosofía m/ Estética y Teoría del Arte de la Universidad de Chile. Luego, en junio de 2018, fue presentado en Buenos Aires en el marco del Foro Analítico del Río de la Plata (FARP).
} 
¿En qué momento surge el vacío en la filosofía? ¿Cuándo emerge como un objeto filosófico digno de reflexión? Bajo el telón de fondo de estas preguntas propongo una tesis inicial para comenzar este trabajo: en el principio, quizás ahora también, la filosofía intentó liberarse del problema del vacío; de esta manera, desde el inicio la filosofía intentó expulsar y desterrar al vacío de su reflexión. Por ejemplo, si decidimos tomar el llamado "Poema ontológico" de Parménides como el comienzo apropiado de la reflexión filosófica, observamos en él una elección clara entre dos caminos: "Pues bien yo te diré - cuida tu de la palabra escuchada - las únicas vías de indagación que se echan a ver. La primera, que es y que no es posible no ser, de persuasión es sendero (pues a la verdad sigue). La otra, que no es y que es necesario no ser, un sendero, te digo, enteramente impracticable" (Velásquez Ortiz 2010 113). Aquí vemos una entrada "espectacular" de la filosofía occidental respecto al problema del vacío, entrada inicial de la reflexión filosófica que en boca de una diosa que desciende a la tierra logra realizar el logos, lo que es; no obstante, más allá de esta grandilocuente puesta en escena, advertimos una afirmación crucial respecto al ser y su telón de fondo: esta afirmación del ser en filosofía, es mi hipótesis aquí, se realiza debido a la amenaza penetrante del vacío. Si advertimos con atención el poema de Parménides, no intenta evidenciar ahí la existencia del ser, sólo existe la advertencia de la amenaza del camino del vacío y, por esta razón, nos llama a aferrarnos al primero. Observamos aquí una especie de prisa o, como dirá Lacan tiempo después en su texto El tiempo lógico y el aserto de certidumbre anticipada (1945), la necesidad de afirmar el ser antes que el vacío nos atrape: es un estado de emergencia ontológico.

En el contexto del "poema ontológico", la elección por el vacío parece tan inverosímil que no se nos permite imaginar siquiera que alguien se sienta atraído hacia ella, es un camino que no se puede ni se debe adoptar en filosofía. Entonces, la afirmación filosófica a favor del ser es la decisión enfática de evitar el vacío, el agujero, para expulsarlo. Si observamos con mayor atención esta idea, la afirmación filosófica es una afirmación política que da cuenta de un campo de batalla: el ser es un arma que se debe usar contra un enemigo insondable. El logos, como instrumento fundamental en esta batalla, debe ser afirmado constantemente para que no caiga en la trampa de la negatividad, del no-ser, de la producción del vacío: el ser, para los filósofos, es una cuestión de voluntad y elección. Entonces me pregunto nuevamente, ¿Cómo surge el vacío como objeto digno de la reflexión filosófica? Me parece que el atomismo es el primer discurso filosófico en introducir la importancia del vacío, ya no como una alternativa marchita tal como Parménides encuadraba en su poema, sino como un camino ineludible para estar "siendo", expresando así la importancia fundamental del movimiento. Es quizás por este motivo que se suele tomar al atomismo como la primera aparición del materialismo en la historia de la filosofía: en otras palabras, el atomismo nos 
muestra por primera vez que el vacío, el movimiento y el materialismo conviven juntos.

Bajo este contexto, concebimos al atomismo como una reacción contra el campo de batalla de la afirmación del ser propio del poema de Parménides, nos incita a no tomar esa elección obligada de la filosofía y promueve, de una u otra manera, asumir paradójicamente ambas elecciones en conjunto. Pero, ¿cómo es posible que dos caminos que se postulan como mutuamente excluyentes puedan ser recorridos al unísono? Es un problema que también encontraremos con el dualismo de la ontología aristotélica y que, como desarrollaremos más adelante, el atomismo se encarga de diluir.

Lo diremos de este modo simple por ahora: el atomismo introduce el vacío como el componente esencial del ser. Esta idea, en tanto disuelve el dualismo filosófico clásico, ha redefinido toda filosofía materialista contemporánea: la concepción de vacío suscita cualquier movimiento ético-político que intente promover nuevos seres. Si volvemos a la filosofía eleática de Parménides veremos que esta se caracteriza por dos principios universales y estáticos: 1) El ser es uno e indivisible; 2) El ser es (no puede no ser) y el no ser no lo es. Al contrario de estos dos principios, el atomismo adoptó el siguiente punto de vista: el ser es divisible por uno ¿Qué quiere decir esto? El atomismo postula al átomo como la partícula indivisible en donde todo lo demás -el universo, el mundo, los "seres"- se puede descomponer imponiendo, como consecuencia, facetas diversas e infinitas del ser. El átomo es el uno sin posibilidad de descomposición adicional, todo lo que puede ser es reducido a este conteo infinito del uno mostrando, más allá del universal de Parménides, la particularidad del uno; en segundo lugar, en el momento que se postula a este uno como la partícula elemental del ser, al unísono, se postula el vacío que separa a los átomos y que permiten, esta es la novedad de tomar los dos caminos del vacío y el ser, el movimiento y la creación. Por lo tanto, para que exista movimiento, se postula el "no ser" en el centro del "ser", esta es la tesis atomista en donde el "no ser es". Podemos reducir lo recién expuesto a dos principios radicalmente contrarios a la filosofía de Parménides: la particularidad del uno y el movimiento de ellos en el vacío.

De esta manera, si concebimos alguna división en los átomos, lo que se divide no es a ellos mismos sino al vacío que los rodea y les permite ser contados infinitamente en su particularidad. El principio de Parménides se basa en un Uno estático como denominador común de todo ser, el de la totalización, mientras que el principio del atomismo es el de la división y movimientos, por este motivo, introduce una grieta en el ser que no se puede totalizar en el Uno al escindirlo radicalmente. Por lo tanto, tomando en serio la posición materialista del atomismo en esta batalla, debemos incluir al vacío como la otra mitad faltante del ser del átomo: el materialismo emerge de una grieta que muchos filósofos por pánico, 
defensa y necesidad de exclusión han querido suprimir. Incluso de una manera precisa y paradójica: usando, en el campo de batalla político, al enemigo como aliado ¿No ha evitado uno el vacío abrazándolo? La filosofía, dentro de esta estrategia, si no ha logrado suprimir el vacío, al menos lo ha hecho manejable, contable.

No pretenderemos hacer aquí lo mismo, por esta razón acudiremos a la expresión imprescindible que da cuenta del movimiento entre átomos en el vacío: clinamen, término utilizado por Lucrecio en De rerum natura. Lucrecio indica que los átomos, las partículas indivisibles, están dotados de peso por su principio de movimiento en el espacio; en consecuencia, todos caen a la misma velocidad, señala así que el movimiento de los átomos es análogo al de las gotas de lluvia: "imbrisutiguttae caderent inane profundum" o "todo caería hacia abajo como gotas de lluvia a través del profundo vacío" (Lucrecio 2003 186). Sin embargo, Lucrecio señala que con solo este gesto nunca surgiría nada en el universo, necesariamente tiene que haber una desviación, un viraje, una declinación del movimiento descendiente que provoqué encuentros y colisiones entre los átomos: recién, con esta desviación, se produce el universo como lo conocemos. Es interesante señalar aquí que Lucrecio, paradójicamente, mantiene tres condiciones para el clinamen: en primer lugar, la declinación ocurre en un lugar no determinado, no tiene lugar o momento asignado, se encuentra desplazado, desfasado, desarticulado del espacio; en segundo lugar, es expresamente mínimo, la declinación es la más leve que puede concebir," nec plus quam mínimum" o "no más que el mínimo posible" (Lucrecio 2003 186) y, por último, Lucrecio se desvía de su argumento cosmogónico y se sumerge en una discusión sobre el libre albedrío; en otras palabras, y es lo que nos interesa en este trabajo, la causalidad de la naturaleza da paso a la causalidad de la cultura - cuestión que recuerda el texto "Sobre la causalidad psíquica" de Lacan: así como los átomos declinan de su camino en su movimiento de creación, también lo hace nuestra voluntad humana que declinará de la determinación de los lazos con la necesidad, el destino, el placer. Lo interesante de toda esta reflexión de Lucrecio es que con la desviación del movimiento propio del clinamen se abre la posibilidad de pensar por vez primera, y de forma materialista, la libertad: en específico, la libertad sería aquí el romper con la universalidad del destino. El clinamen de Lucrecio es el punto donde el cosmos y la humanidad, superponiéndose, muestran con el movimiento en el vacío un fuera de lugar y fuera de tiempo radicalmente subversivo en la historia de la filosofía.

Si seguimos en la batalla filosófica alrededor del ser y el vacío encontramos, por ejemplo, que el gran crítico del clinamen fue Cicerón en su De fato: "esto es completamente una ficción infantil (...) por un lado es esta declinación arbitrariamente soñada (Epicuro dice que el átomo declina sin causa; para un físico no hay nada más vergonzoso (nihil turpius) que decir que algo 
sucede sin causa" (Schmidt 2007 46). No obstante, más allá de Cicerón, encontramos otras resistencias y críticas al clinamen en la batalla de la filosofía: Plutarco, Plotino, Agustín, Kant hasta Hegel. Por ejemplo, Kant en su Historial general de la naturaleza y teoría del cielo señala que "Epicuro fue aún más descarado que recurrió a los átomos que declinaran su movimiento recto sin ninguna causa para que puedan encontrarse" (Kant 1946 234). Luego encontramos a Hegel, quién trato a esta noción con desprecio al señalar en su Historia de la filosofía que para Epicuro los átomos se desvían del movimiento recto "en una línea curva (en einer krummen line) que se aparta de la dirección recta, de modo que colisionan con cada otro, formando así una unidad meramente superficial (eine oberflächliche Einheit) no derivada de su esencia" (Hegel 2001 313).

Lo interesante de estas afirmaciones de la filosofía respecto al movimiento y el vacío es que muestran que la noción de clinamen refiere a algo inherentemente irreflexivo, superficial, externo al pensamiento y a la deducción conceptual. De esta manera, llegamos al fondo del problema que intentamos plantear en este trabajo: ¿Qué pertenece a la esencia del átomo? ¿Es el clinamen una adición externa meramente superficial que no afecta su esencia? ¿Es un desvío sin razón suficiente? ¿Es inherente o externo al destino del átomo? Para debatir con los puntos de vista críticos sobre el clinamen debemos recurrir a Deleuze en el apéndice sobre Lucrecio de La lógica del sentido: "Clinamen o declinación no tiene nada que ver con el movimiento oblicuo que vendría a modificar por accidente una caída vertical. Está presente desde siempre: no es un movimiento secundario ni una determinación secundaria del movimiento que ocurriría en cierto momento, en un lugar en particular. El clinamen es la determinación originaria de la dirección del movimiento del átomo" (Deleuze 2017 311).

Para seguir con la línea de pensamiento de Deleuze, pensamiento que va en contra de todo el grano de la tradición filosófica que acabamos de señalar, diremos que el clinamen siempre está ahí, es la disparidad arraigada desde el "origen" en la definición de átomo, no es un mero accidente al ser, es una disparidad inherente a sí misma: en otras palabras, el átomo es su propia declinación. No es meramente la unidad paradójica de uno y el vacío sino, al unísono, la unidad de la entidad con el propio alejamiento de sí misma. Por lo tanto, a diferencia de la tradición filosófica, Deleuze nos muestra que el clinamen no es un destino secundario que le sobreviene al camino recto del átomo; por el contrario, uno supone ilusoriamente un segundo momento de desvío donde en principio habría una dirección recta, pero esta dirección no existe en sí misma, la narrativa temporal que coloca las cosas en secuencias - primero el caer, luego la desviación- es una ilusión retroactiva. De esta manera, un Deleuze materialista nos permite pensar que la declinación habita en los átomos desde el principio, y habita de forma permanente, en todo momento. 
El fuera de lugar y fuera de tiempo del clinamen es parte inherente del lugar y el tiempo del átomo: el clinamen es el "alma" materialista del átomo. Podríamos arriesgar decir que incluso se vuelve su "esencia accidental", la manera en que la esencia puede ser ella misma exponiendo radicalmente su contingencia: la unidad del átomo está de por sí desviada, por estructura. Por lo tanto, Deleuze advierte el punto político-económico fundamental del materialismo del clinamen, pero podríamos también acudir a la tesis doctoral del joven Karl Marx titulada como La diferencia entre la filosofía de la naturaleza de Demócrito y Epicúreo (1841) donde refiere a algo similar y fija, al mismo tiempo, el destino de su propia filosofía en la noción de clinamen. No opinaremos aquí sobre la línea fundamental de este trabajo de Marx, para muchos cuestionable, pero nos ocuparemos de su decisión de oponerse a la tradición filosófica que criticaba duramente al atomismo al mantener, precisamente, que el clinamen es inherente al átomo y no externo: "Cicerón lamenta aún más (...) que la declinación del átomo ocurra sin una causa; y nada más vergonzoso, dice Cicerón, le podría ocurrir a un físico. Pero, primero, una causa física como la que busca Cicerón arrojaría la declinación del átomo de nuevo en la serie determinista de la que debe precisamente levantarse. Y, además, el átomo aún no se ha logrado antes de ser determinado por la declinación. Para cuestionar la causa de esta determinación, por lo tanto, significa cuestionar a la causa que convierte a un átomo en un principio" (Marx 1971 282).

¿Cuál es la causa de la causa? ¿Tiene la causa una causa? ¿Qué se requiere de una causa para que sea un principio? ¿Puede una causa cojear? El argumento de Marx es básicamente que, una vez que postulamos al átomo como principio, no hay otra causa que lo pueda afectar aparte de la causa ya inscrita en él, la declinación pertenece a su causa inherente, no a su destino posterior: es, precisamente, la disparidad inherente de la uniformidad y la univocidad. Esto nos recuerda nuevamente a Lacan cuando indica que la causa "de ce qui cloche" o "la causa coja", siempre conviviendo con cualquier causa.

La política materialista del clinamen que Deleuze y Marx advirtieron explícitamente, se opone así a algunos dualismos básicos de la ontología aristotélica que se asumen espontáneamente en la generalidad filosófica. El átomo no es hylé ni morphe, ni materia ni forma, es un principio que evade tal división y todas las complicaciones del hylomorphismo aristotélico. El átomo es ambos, materia y forma en "uno", no requiere a la forma separada de la no-forma, es la no-forma propulsada por sí misma, dotada de su propio impulso e ímpetu al igual que su movimiento recto-desviado. Lo simple, y al mismo tiempo difícil, de este atomismo es su contrapelo con las dualidades aristotélicas que nos parecen evidentes; la manera de evadir esto para pensar "los dos en uno" - no un Uno absoluto - es la siguiente tesis: la desviación interna de la unidad, desmantela la unidad. Esta es la herramienta atomista en el campo de batalla filosófico. 
Ahora, en el desarrollo de esta trabajo advertimos dos posibilidades contemporáneas de leer la subversiva tesis del clinamen: la primera sitúa al clinamen en el lugar de una excepción constitutiva, algo debe haber ocurrido para que el universo emerja, ocurriendo fuera del espacio y tiempo del universo, sin lugar en ellos cuando se encuentran ya constituidos; la segunda, bien puede ser pensado como principio "cuasi universal" omnipresente, principio que descarrila inmanentemente cualquier universalidad en todos los espacios y tiempos. La primera opción es explicitada por Badiou: “Es absolutamente necesario que el clinamen sea a su vez abolido (...) Cualquier explicación particular no requiere clinamen, aunque la existencia de cualquier cosa en general es impensable sin ella (...). El átomo afectado por desviación engendra el Todo sin ningún resto o rastro de ese afecto" (Badiou 2008 79- 80). La segunda opción la observamos con Deleuze, y podemos leer su noción de lo virtual como el clinamen inherente e inmanente habitando en cada momento y entidad. El caso de Badiou lo tomaremos como una excepción que nunca "tiene lugar", aunque subyace a toda toma de lugar como una "trascendencia inmanente" fuera del mundo. El caso de Deleuze lo tomaremos como una cuasi-universalización de la excepción, principio que se hace inmanente a cualquier toma de lugar; por lo tanto, la excepción se convierte en un cuasiuniversal que se obstina a destituir cualquier universal, al instituir el fuera de lugar de cualquier Uno ¿Cómo pensar estas dos opciones en conjunto? ¿Acaso existe una tercera opción?

Me permitiré volver a Demócrito y tomar una tercera opción en este campo de batalla que no había sido puesta en consideración, un punto oscuro y crucial que fue señalado por Lacan donde vio algo así como "un átomo de pensamiento y ser". En su seminario titulado Los cuatro conceptos fundamentales del psicoanálisis dice lo siguiente: "Cuando Demócrito trató de designarlo (presentándose a sí como el adversario de una pura función de negatividad para introducir el pensamiento) nos dice: "lo esencial no es el meden (no-ser), y agrega (...) no es un meden, es un den, que, en griego es una palabra fabricada. No dijo uno, y no mencionemos el ser ¿Qué dijo? Dijo - respondiendo a la pregunta que nos formulamos hoy, la del idealismo- ¿Nada, quizás? No - quizás nada, no nada" (Lacan 2011 71).

Entonces, ¿Qué nada es un den? En el fragmento 156 Demócrito enigmáticamente introduce algo que no calza ni en el uno ni en el vacío: lo llamó den. Esto causó problemas a los filósofos clásicos debido a qué es una palabra impropia del griego, una palabra fabricada. Esta expresión muestra la "negación" particular del hen, uno. Este último, hen, se puede negar de dos maneras clásicamente en griego: la primera con ouden (negación de la intención); la segunda con meden (negación subjetiva). Ambos significan "nada" o "no uno"; no obstante, den, esta palabra fabricada por Demócrito, significa "menos de uno, pero aún no es nada", o forzándola un poco, "menos que nada". Esto presenta un hueso duro de 
roer para los traductores; de esta manera, por ejemplo, la brillante Bárbara Cassin propone al den como "menor que nada" tal como Lacan lo señalara en su seminario con su "no nada": "El átomo no es sólo una afirmación o posición, ser o uno, pero además tampoco es su negación, esto es que no tiene la consistencia de no-cosa: el átomo es literalmente menor que nada (...) Den es el nombre del átomo" (Badiou \& Cassin 2011 81).

Entonces cabe preguntarse nuevamente, ¿Qué es esta entidad llamada den? No es algo, no es nada, no es, no es uno, no es positivamente existente, no es ausente, no es contable, “¿Nada, quizás? No - quizás nada, no nada”. Creo que esta frase de Lacan nos muestra una clave: el den no es una negación, sino más bien una "decapitación de la nada", no es una sustracción del ser que introduce un vacío, ni tampoco es un sobrante de la negación del ser que no tuvo éxito; por el contrario, el den es, paradójicamente, "una sustracción del no-ser": en otras palabras, es la negatividad envaneciéndose a sí misma. Por lo tanto, algo emerge en el den, en el fracaso de la negatividad, pero no emerge del todo debido a que no tiene positividad o identidad. A pesar de esta condición particular, den es el ser del átomo.

Lo interesante de toda esta reflexión, es que Demócrito nos muestra que los átomos, den, no son simplemente seres positivos implicados en el vacío, sino que más bien se encuentran constantemente "entre" un espacio de ser y no-ser. Ahora, y consecuencia de esto, si el átomo es den, para Demócrito este último no puede tener peso -diferencia radical con Lucrecio y Epicuro-; es decir, para Demócrito no puede haber caída paralela en el clinamen. Los átomos, el den, entonces no son cuerpos con peso, sino trayectoria, movimiento, que producen como efecto de ello cuerpos. Para tener claro esto, Demócrito habla del rhysmos, las ondas en movimiento y sin peso como propiedad básica de los átomos.

A medida que vamos desarrollando estas ideas, advertimos que den es un escándalo ontológico. Lacan en El Atolondradicho dirá que: "Demócrito nos ha hecho el regalo de los átomos, de lo real radical, de elidir su "no", me, pero en su subjuntividad, o sea, ese modal cuya demanda vuelve a hacer consideración. Gracias a lo cual el den fue justamente el pasajero clandestino cuyo clam hace ahora nuestro destino. No más materialista en eso que cualquiera que fuese sensato, yo o Marx" (Lacan 2012 518).

El problema del clinamen es indicativo de la batalla de la filosofía respecto al vacío, el ser y el materialismo. Por ejemplo, vimos que Hegel hablaba despreciativamente del clinamen poniéndose del lado de sus detractores; por otro lado, Deleuze realizó una formulación formidable del clinamen apoyando la causa atomista de Lucrecio al posicionarlo explícitamente en una política materialista. Por último, con Lacan seguimos el invento de Demócrito del den como sustracción de la negatividad: esta posición evita postular al clinamen como una excepción 
constitutiva (Badiou) o una universalizada virtualidad (Deleuze) al producir un nuevo objeto que, sin embargo, yace materialistamente bajo desde los albores de la filosofía como su pasajero clandestino: un objeto sin identidad y fundador de una no ontología. Este es quizás el lugar que puede ocupar el psicoanálisis en la discusión materialista: mostrar aquel objeto, objeto a, como una nueva herramienta en la batalla de la filosofía.

\section{Bibliografía}

Badiou, Alain y Cassin, Bárbara. No hay relación sexual. Madrid: Amorrrortu, 2011. Badiou, Alain. Teoría del Sujeto. Buenos Aires: Prometeo, 2011.

Deleuze, Gilles. Lógica del sentido. Barcelona: Paidós, 2007.

Hegel, George. Wilhem. The Philosophy of History. Ontario: Batoche books, 2001.

Kant, Imanuel. Historial general de la naturaleza y teoría del cielo. Buenos Aires: Lautaro, 1946.

Marx, Karl. La diferencia entre la filosofía de la naturaleza de Demócrito y Epicúreo. Madrid: Ayuso, 1971.

Lacan, Jacques. Los cuatro conceptos fundamentales del psicoanálisis. Buenos Aires: Paidós, 2011.

Lacan, Jacques. El atolondradicho. Buenos Aires: Paidós, 2012.

Lucrecio. De la naturaleza de las cosas, Buenos Aires: Biblioteca Virtual Universal, 2003.

Schmidt, E.A. Clinamen, Heidelberg: Universitatsverlag Winter, 2007.

Velásquez Ortiz, Sonia. "De multiplicidades y contradicciones: la poesía de Oliverio Girondo". Hojas Universitarias. Universidad Central de Colombia 63 (2010): 106-121. 